\title{
Vibrations of a chain of Xe atoms in a groove of carbon nanotube bundle
}

\author{
Marko T. Cvitaš* \\ Department of Chemistry, University of Durham, South Road, Durham DH1 3LE, UK \\ Antonio Šiber ${ }^{\dagger}$ \\ Institute of Physics, P.O. Box 304, 10001 Zagreb, Croatia
}

\begin{abstract}
We present a lattice dynamics study of the vibrations of a linear chain of Xe adsorbates in groove positions of a bundle of carbon nanotubes. The characteristic phonon frequencies are calculated and the adsorbate polarization vectors discussed. Comparison of the present results with the ones previously published shows that the adsorbate vibrations cannot be treated as completely decoupled from the vibrations of carbon nanotubes and that a significant hybridization between the adsorbate and the tube modes occurs for phonons of large wavelengths.
\end{abstract}

Published in Phys. Rev. B 67, 193401 (2003)

Adsorption of gases in carbon nanotube materials has recently attracted considerable attention. ${ }^{1-6}$ Studies of this phenomenon are in part motivated by the suggested application of carbon nanotubes for an efficient gas storage. ${ }^{7}$ Theoretical studies of adsorption in carbon nanotubes have predicted unique, one-dimensional (1D) phases of the adsorbed gases. ${ }^{1,3,5}$ The dimensionality of the adsorbate structures is reduced due to a specific geometry of an isolated carbon nanotube and bundles (or ropes) of carbon nanotubes. This opens a possibility to use nanotubes as a template for growth of adsorbed nanostructures such as atomic and molecular "wires".

The nanotube materials commonly studied and produced do not contain isolated carbon nanotubes, but the socalled bundles of nanotubes, containing $\sim 100$ nanotubes organized in a triangular lattice. ${ }^{9}$ The bundle surface can support 1D phases of matter. ${ }^{3}$ The potential for adsorption of gases on the bundle surface is stronger in the region between the two tubes (groove) than in other positions. ${ }^{6}$ Thus, the atoms bound in these positions should desorb from the surface at higher temperatures than those adsorbed at other places, which clearly suggests that the 1D phases of matter adsorbed in the groove positions can be prepared by carefully programmed thermal desorption.

The detection of such phases by specific heat measurements has been suggested in Ref. 5 and their signature in the low-temperature specific heat has been theoretically predicted. The approach of Ref. 5 was based on two simplifying assumptions: (i) the discreteness of the carbon nanotube was neglected and (ii) the nanotubes were treated as completely rigid, providing a static external potential for the adsorbed atoms. The adsorbate vibrational modes obtained in that study were completely localized on the adsorbate atoms due to the neglect of coupling between the adsorbate and substrate vibrations. As the low-temperature thermodynamics of adsorbates crucially depends on the details of the excitation spectrum, more realistic model of adsorbate vibrations is needed in order to predict and interpret correctly measurements of thermodynamical quantities such as specific heat.

In this article, we present calculations of the phonon dispersions and phonon eigenvectors of a 1D phase of Xe atoms adsorbed in a groove of carbon nanotube bundle. Unlike in the previous studies focused on adsorption of gases ${ }^{1-6}$, we do not treat the nanotubes as rigid and smooth objects which enables us to examine the effects of hybridization of adsorbate and substrate vibrations and to calculate the phonons of the realistically modeled system.

Due to a large radius of Xe atoms the occupation of interstitial channel positions is not possible. For nanotubes with closed ends, the adsorption in the tubes is also not possible. Thus, for low coverage and pressure, only the groove positions are expected to be occupied. The system we examine is represented in Fig. 1. It consists of two infinitely long $(17,0)$ single wall carbon nanotubes with a Xe chain in between them. The two tubes are mirror images of one another and their axes are separated by $2 R+3.2 \AA$, where $R$ is the tube radius $[R=6.6 \AA$ for $(17,0)$ tube; note that the diameter of $(17,0)$ nanotube is close to mean diameter of carbon nanotubes in the samples studied experimentally $\left.{ }^{10}\right]$. For this particular, highly symmetric system, Xe phase commensurate with the tubes is expected to form in a range of temperatures, similarly as for Xe monolayers on graphite surface. ${ }^{11}$ The commensurability of this phase is the main reason for its choice in the present study, since the periodicity of its structure simplifies the calculations. Such a phase

\footnotetext{
*E-mail: m.t.cvitas@durham.ac.uk

${ }^{\dagger}$ E-mail: asiber@ifs.hr
} 
is unlikely to exist in a realistic situation, where a range of finite axial and angular offsets of the two neighboring tubes are to be expected. Nevertheless, the coupling between the adsorbate and substrate vibrations persists even in an incommensurate adsorbate phase ${ }^{12}$, which may be expected in the case of a poor alignment of the adjacent two tubes. This coupling can be easily studied for a commensurate phase represented in Fig. 1 where a lattice dynamics approach $^{13}$ is possible. The general features of the coupling between the adsorbate and substrate vibrations are expected to be similar in incommensurate and commensurate phases, based on previous experimental and theoretical evidence for such phases of adsorbate monolayers on crystal surfaces. ${ }^{12,14,15}$. The fact that we consider only two tubes defining a groove region is not of importance, since the interaction of Xe atoms with more distant tubes in the bundle is very small due to large $\mathrm{Xe}-\mathrm{C}$ distances for $\mathrm{C}$ atoms forming these nanotubes.

The nearest neighbor Xe-Xe distance in the phase depicted in Fig. 1 is $3 a=4.26 \AA$, where $a$ is the nearest neighbor $\mathrm{C}-\mathrm{C}$ spacing. The position of a single Xe adsorbate was found by the potential energy minimization procedure, using Xe-C binary potential suggested in Ref. 2, also used in Ref. 5. As a result of this procedure, we obtained that the height of the Xe chain above the plane passing through the tube axes is $6.3 \AA$. The structure we study can be obtained by one-dimensional discrete translations of a unit cell containing $136 \mathrm{C}$ and one Xe atom. The vibrations of isolated carbon nanotube were modeled using a set of force constants for intraplanar interactions in graphite developed in Ref. 16. As noted by $\mathrm{Lu}^{17}$, the intertube interactions must be given a special treatment, and for these interactions we have adopted the approach of Ref. 17, i.e. we introduced an effective binary C-C potential of Lennard-Jones (LJ) form with the parameters $\epsilon_{C-C}=12 \mathrm{meV}$ and $\sigma_{C-C}=3.4 \AA$. Xe-Xe interactions were modeled by LJ pair potential with parameters $\epsilon_{X e-X e}=19.07 \mathrm{meV}$ and $\sigma_{X e-X e}=4.1 \AA^{2}{ }^{2}$ More refined Xe-Xe potentials exist ${ }^{18}$, but we choose the one employed in Ref. 5 in order to examine the effects of approximations introduced in that work. The results of the calculation with more precise Xe-Xe potential will be discussed later.

The calculated phonon dispersions, $\omega(q)$, where $\omega$ and $q$ are the phonon frequency and wave vector, and the corresponding density of states, $\rho(\omega)$, are presented in Fig. 2. The density of states is calculated as

$$
\rho(\omega)=\sum_{i} \sum_{q} \delta\left[\omega-\omega_{i}(q)\right]
$$

where the index $i$ labels the different phonon branches. In plotting this quantity, we have represented the $\delta$-functions in Eq. (1) with normalized Gaussian functions with width parameters of $0.3 \mathrm{meV}$ [panel (b)] and $0.15 \mathrm{meV}$ [panel (d)]. The high energy portion of the phonon spectrum (panels (a) and (b) of Fig. 2) correlates quite well with the spectrum of isolated nanotube studied in Refs. 19-21. At frequencies between 0.8 and $6.0 \mathrm{meV}$, the adsorbate vibrations dominate the phonon density of states and the peak in the density of states (denoted by arrow in panel (b) of Fig. 2), not originating from the tube vibrations is clearly seen. The low frequency region of the dispersion curves and the density of states is shown in panels (c) and (d) of Fig. 2. The three adsorbate induced modes denoted by $L, T 1$ and $T 2$ are clearly visible. To examine the polarization of these modes, we plot in Fig. 3 the quantity defined as

$$
\rho_{\nu}^{X e}(\omega, q)=\sum_{i}\left|e_{i, \nu}^{X e}(q)\right|^{2} \delta\left[\omega-\omega_{i}(q)\right], \nu=x, y, z,
$$

where $e_{i, \nu}^{X e}(q)$ is the $\nu$-th Cartesian component of the polarization vector of the $i$-th phonon branch at the position of a Xe atom. The quantity in Eq. (2) is directionally and wave vector resolved density of Xe vibrations.

One can observe in Fig. 3 that the mode denoted by $L$ corresponds to vibrations of the Xe chain polarized along $x$ direction i.e. along the chain direction (longitudinal mode), while the modes denoted by $T 1$ and $T 2$ correspond to vibrations of Xe atoms in $y$ and $z$ directions, respectively (transverse modes). The dashed lines in panel (c) of Fig. 2 represent the dispersions of $\mathrm{L}, \mathrm{T} 1$ and T2 modes obtained in Ref. 5 . Note that in contrast to Ref. 5 the $L$-mode acquires a gap $(\sim 0.8 \mathrm{meV})$ at the center of the quasi one dimensional Brillouin zone which is a consequence of the commensurability of the Xe chain with the substrate. A similar effect has been observed for commensurate Xe overlayers on crystal surfaces. ${ }^{15,22,23}$ While the $L$ mode does not hybridize with the tube modes, the two transverse adsorbate modes are influenced by the elasticity of the tube substrate which is clearly seen for wave vectors smaller than about $0.11 / \AA$. In this long-wavelength region, the adsorbate atoms vibrate with several frequencies determined by the details of the nanotube phonon spectrum. The vibrations of Xe atoms in this region cannot be treated as vibrations in which only Xe atoms participate, since the atoms of the two substrate tubes are also involved in these vibrations. As a result, the localization of these modes on Xe atom is smaller from the corresponding value at the end of the Brillouin zone $(q=0.7371 / \AA)$, where the $L, T 1$, and $T 2$ modes represent $100 \%$ pure Xe vibrations in which the substrate atoms do not participate. The adsorbate-substrate mode mixing appears only in the long wavelength region due to large "stiffness" of the nanotube phonons when compared to adsorbate modes. Thus, the modes cross only for small q values. 
Examination of the polarization vectors of all atoms in the unit cell reveals that the motion of carbon atoms in the region of hybridization is highly complex. The displacements of $\mathrm{C}$ atoms from their equilibrium positions have in general all three Cartesian components. Transforming these components into cylindrical coordinate system does not lead to much more insight since the modes are again highly complex and have radial, tangential and $z$-components which are all different from zero. However, it can be said that the radial components of the polarizations vectors of $\mathrm{C}$ atoms slightly dominate the motion pattern.

We find that the frequencies of the two transverse phonons agree reasonably well with the results published in Ref. 5. The differences can be attributed to the facts that in Ref. 5 the two tubes were treated as smooth and that the tubes considered in Ref. 5 were of somewhat larger radius $(6.9 \AA)$ than the ones studied in this work $(6.6 \AA)$. As in Ref. 5, we find that the adsorbate transverse vibrations polarized in the $z$-direction exhibit lower frequencies than those polarized in the $y$-direction. This is a consequence of a specific shape of the potential experienced by Xe adsorbate in the groove (Fig. 2 of Ref. 5).

The maximum frequency of the $L$-mode obtained in this study is almost twice larger than the one obtained in Ref. 5 . The reason for this is that the nearest neighbor Xe-Xe radial force constant is significantly larger in the commensurate phase than in the incommensurate phase studied in Ref. 5. This is due to smaller Xe-Xe distances dictated by the corrugation of the underlying substrate (bundle of tubes).

Use of more realistic Xe-Xe potentials ${ }^{18}$ results in lower frequency of the $L$-mode at the Brillouin zone boundary. Using the HFDB2 Xe-Xe potential suggested in Ref. 18, we obtain the $L$-mode frequency at the zone edge of 4.62 $\mathrm{meV}$, while the $L$-mode zone center gap does not change $(0.8 \mathrm{meV})$. The two transverse modes retain practically the same dispersions as those depicted in Fig. 2. This is not surprising, since the transverse mode frequency is determined mainly by Xe-C interactions. Renormalization of the Xe-Xe interactions by the substrate ${ }^{24,25}$ results in a modified effective Xe-Xe potential. In the study of these effects reported in Ref. 24, it was found that the interaction between the two Xe atoms adsorbed in the groove positions is weaker than in free space and that it can be approximately represented by a scaling of the energy parameter of the LJ Xe-Xe potential. ${ }^{24}$ Applying an analogous scaling to the HFDB2 potential, we obtain further reduction of the maximum $L$-mode frequency, which now amounts to $4.05 \mathrm{meV}$ (at the zone edge).

It is of interest to compare the present results with the theoretical and experimental results for vibrations of the commensurate $(\sqrt{3} \times \sqrt{3}) \mathrm{R}^{\circ} 0^{\circ}$ phase $^{11}$ of monolayer of Xe atoms on graphite. The arithmetic mean of the zone edge frequencies of the two transverse modes in the present system is quite close to the measured frequency of the transverse mode (with polarization perpendicular to the surface) of a Xe monolayer on graphite ${ }^{23}(\approx 3 \mathrm{meV})$. Molecular dynamics calculations for commensurate monolayer phase of Xe on graphite ${ }^{26}$ yielded $L$-mode dispersion with maximum frequencies of $5.6 \mathrm{meV}$ ( $\mathrm{M}$ point in the surface Brillouin zone) and $4.85 \mathrm{meV}$ ( $\mathrm{K}$ point in the surface Brillouin zone). These values are comparable to the maximum frequency of the $L$ mode we obtain in this work. The zone center gap of $L$-mode was found to be $\sim 0.9 \mathrm{meV}$, also very close to the value we obtained for the Xe chain phase $(0.8 \mathrm{meV})$. Very similar results have been obtained in the lattice dynamics study of a Xe monolayer on graphite in Ref. 27. Some points in connection with this comparison should be emphasized, however. Whereas the two transverse modes are practically dispersionless in the chain phase we study, the in-plane transverse vibration of Xe monolayer on graphite exhibits a large dispersion. ${ }^{26,27}$ This is a direct consequence of quite different geometries of the substrate material in the case of adsorption on a planar graphite and on the bundle of carbon nanotubes. Additionally, although the characteristic phonon frequencies are similar for Xe/graphite and Xe/groove systems, one has to remember that the dimensionality of the phonon phase space is different in the two systems. Wave vector $q$ is a one dimensional quantity in the present case, whereas in the case of Xe/graphite, it is a two dimensional quantity. This has direct consequences on the low-temperature thermodynamics of adsorbed phases. ${ }^{6}$

In summary, we have investigated the vibrations of a commensurate phase of Xe atoms adsorbed in the groove positions of a carbon nanotube bundle. Model we used is significantly improved over previous models which have treated the nanotube substrate as rigid and smooth. ${ }^{1-3,5,6}$ This enabled us to study the details of the adsorbate phonon spectrum and we have shown that a "mixing" between the transverse adsorbate (Xe) and substrate (nanotube bundle) phonon modes can be expected for large phonon wavelengths. Such mixing does not appear for the adsorbate mode with longitudinal polarization. Since the low-temperature thermal properties are dominated by excitations of lowfrequency modes (which is precisely the region where the hybridization occurs), it can be expected that the signature of the adsorbate phase in the overall low-temperature thermal properties of the sample will be more complicated than previously predicted..$^{4-6}$ 
${ }^{1}$ M.W. Cole, V.H. Crespi, G. Stan, C. Ebner, J.M. Hartman, S. Moroni, and M. Boninsegni, Phys. Rev. Lett. 84, 3883 (2000)

${ }^{2}$ G. Stan, M.J. Bojan, S. Curtarolo, S.M. Gatica, and M.W. Cole, Phys. Rev. B 62, 2173 (2000)

${ }^{3}$ M.M. Calbi, S.M. Gatica, M.J. Bojan, and M.W. Cole, J. Chem. Phys. 115, 9975 (2001)

${ }^{4}$ M.M. Calbi and M.W. Cole, Phys. Rev. B 66, 115413 (2002)

${ }^{5}$ A. Šiber, Phys. Rev. B 66, 235414 (2002)

${ }^{6}$ A. Šiber, Phys. Rev. B 66, 205406 (2002)

${ }^{7}$ A.C. Dillon, K.M. Jones, T.A. Bekkedahl, C.H. Kiang, D.S. Bethune, and M.J. Heben, Nature (London) 386, 377 (1997)

${ }^{8}$ G. Gao, T. Cagin, and W.A. Goddard III, Phys. Rev. Lett. 80, 5556 (1998); A. Rubio, Y. Miyamoto, X. Blase, M.L. Cohen, and S.G. Louie, Phys. Rev. B 53, 4023 (1996)

${ }^{9}$ P.M. Ayan and T.W. Ebbesen, Rep. Prog. Phys. 60, 1025 (1997)

${ }^{10}$ E. Anglaret, S. Rols, and J.-L. Sauvajol, Phys. Rev. Lett. 81, 4780 (1998)

${ }^{11}$ G. Bracco, P. Cantini, A. Glachant, and R. Tatarek, Surf. Sci. 125, L81 (1983)

12 B. Hall, D.L. Mills, and J.E. Black, Phys. Rev. B 32, 4932 (1985)

${ }^{13}$ R.E. Allen, G.P. Alldredge, and F.W. de Wette, Phys. Rev. B 4, 1648 (1971)

${ }^{14}$ A.P. Graham, M.F. Bertino, F. Hofmann, J.P. Toennies, and Ch. Wöll, J. Chem. Phys. 106, 6194 (1997)

15 J. Braun, D. Fuhrmann, A. Šiber, B. Gumhalter, and Ch. Wöll, Phys. Rev. Lett. 80, 125 (1998); A. Šiber, B. Gumhalter, J. Braun, A.P. Graham, M. Bertino, J.P. Toennies, D. Fuhrmann, and Ch. Wöll, Phys. Rev. B 59, 5898 (1999)

${ }^{16}$ R. Nicklow, N. Wakabayashi, and H.G. Smith, Phys. Rev. B 5, 4951 (1972)

17 J.P. Lu, Phys. Rev. Lett. 79, 1297 (1997)

18 A.K. Dham, W.J. Meath, A.R. Allnatt, R.A. Aziz, and M.J. Slaman, Chem. Phys. 142, 173 (1990)

${ }^{19}$ J. Yu, R.K. Kalia, and P. Vashishta, J. Chem. Phys. 103, 6697 (1995)

${ }^{20}$ A. Charlier, E. McRae, M.-F. Charlier, A. Spire, and S. Forster, Phys. Rev. B 57, 6689 (1998)

${ }^{21}$ M.S. Dresselhaus, P.C. Eklund, Advances in Physics, 49, 705 (2000)

${ }^{22}$ L.W. Bruch, A.P. Graham, and J.P. Toennies, Mol. Phys. 95, 579 (1998)

${ }^{23}$ J.P. Toennies and R. Vollmer, Phys. Rev. B 40, 3495 (1989)

${ }^{24}$ M.K. Kostov, M.W. Cole, J.C. Lewis, P. Diep, and J.K. Johnson, Chem. Phys. Lett. 332, 26 (2000)

${ }^{25}$ L.W. Bruch, M.W. Cole, and E. Zaremba, Physical Adsorption: Forces and Phenomena (Clarendon Press, Oxford, 1997)

${ }^{26}$ M. Marchese, G. Jacucci, and M.L. Klein, Surf. Sci. 145, 364 (1984)

${ }^{27}$ E. de Rouffignac, G.P. Alldredge, and F.W. de Wette, Phys. Rev. B 24, 6050 (1981)

FIG. 1. Two $(17,0)$ carbon nanotubes with a commensurate Xe chain in between them. This distance ratios in this figure correspond to the ones used in the calculations. Our choice of the coordinate system is also denoted.

FIG. 2. (a) Phonon modes of a system displayed in Fig. 1. (b) Phonon density of states. (c) Low frequency portion of the data presented in panel a). Dashed lines represent L,T1, and T2 modes obtained in Ref. 5. (d) Low frequency portion of the data presented in panel b). The density of states from panel b) is multiplied by a factor of 3 to obtain the data in panel d).

FIG. 3. Contour-like plot of $x$ - (top panel), $y$ - (middle panel), and $z$ - (bottom panel) components of the directionally and wave vector resolved phonon density of states at Xe atom. The density of dots in these plots corresponds to $\left|e_{i, \nu}^{X e}(q)\right|^{2}$, where $\nu=x, \nu=y$, and $\nu=z$ for the top, middle and bottom panel, respectively. The highest density of dots corresponds to $\left|e_{i, \nu}^{X e}(q)\right|^{2}=1$. 


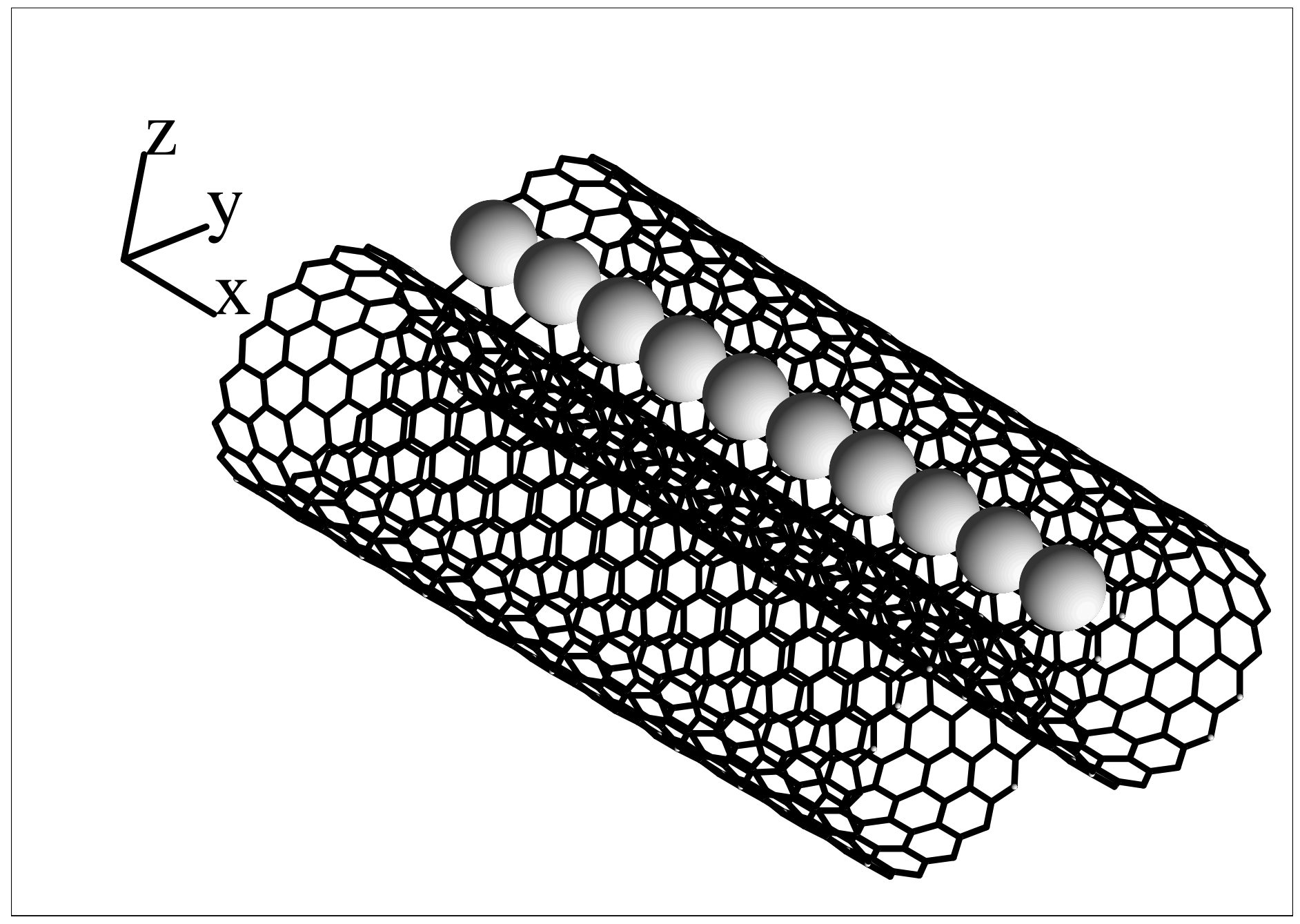




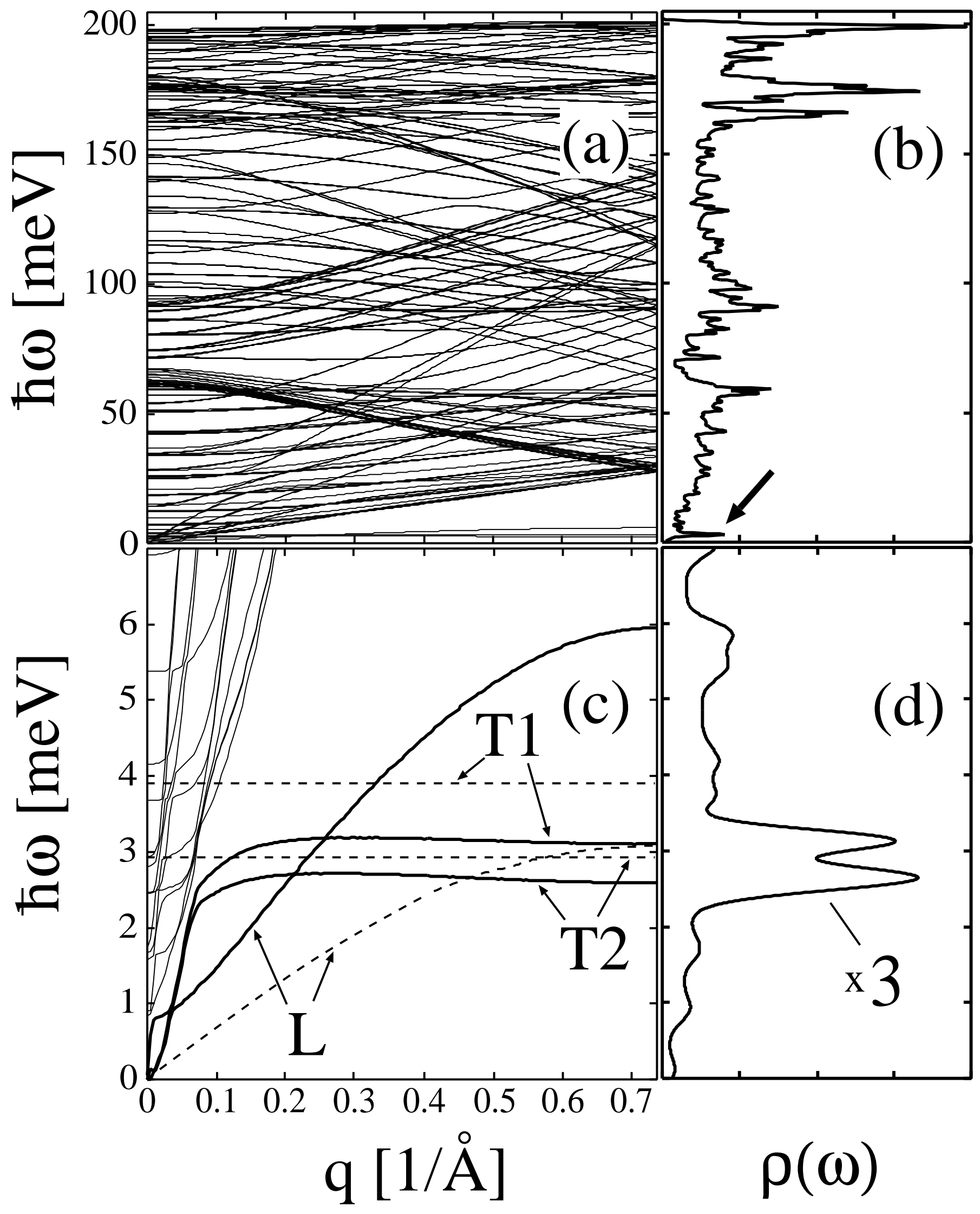




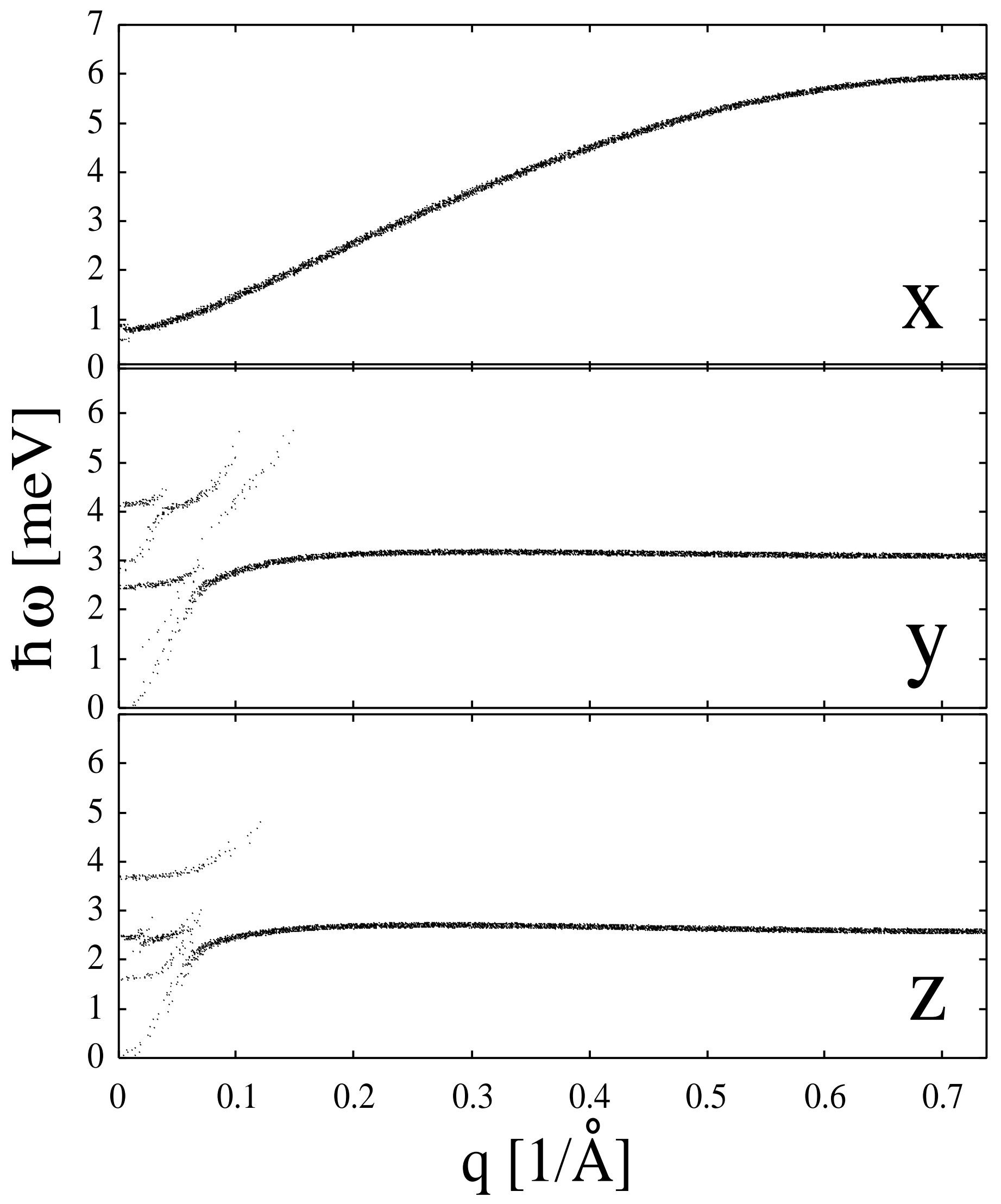

